\title{
Article \\ Electromechanical-Mode Coupling Model and Failure Prediction of CFRP under Three-Point Bending
}

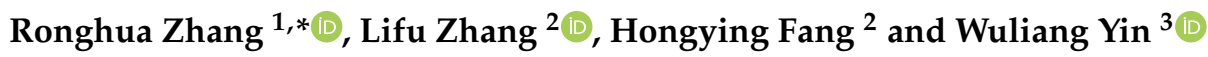 \\ 1 School of Artificial Intelligence, Tiangong University, Tianjin 300387, China \\ 2 School of Control Science and Engineering, Tiangong University, Tianjin 300387, China; \\ zhanglf1997@126.com (L.Z.); hyfang2020@163.com (H.F.) \\ 3 School of Electrical and Electronic Engineering, University of Manchester, Manchester M13 9PL, UK; \\ Wuliang.Yin@manchester.ac.uk \\ * Correspondence: zhangronghua@tiangong.edu.cn
}

check for updates

Citation: Zhang, R.; Zhang, L.; Fang, H.; Yin, W. Electromechanical-Mode Coupling Model and Failure Prediction of CFRP under

Three-Point Bending. Electronics 2021, 10, 2007. https://doi.org/10.3390/ electronics10162007

Academic Editors: Gabriele Grandi, José Matas, Carlos E. Ugalde-Loo and Fushuan Wen

Received: 20 July 2021

Accepted: 15 August 2021

Published: 19 August 2021

Publisher's Note: MDPI stays neutral with regard to jurisdictional claims in published maps and institutional affiliations.

Copyright: (c) 2021 by the authors. Licensee MDPI, Basel, Switzerland. This article is an open access article distributed under the terms and conditions of the Creative Commons Attribution (CC BY) license (https:// creativecommons.org/licenses/by/ $4.0 /)$.

\begin{abstract}
Carbon fiber reinforced polymer materials (CFRP) cause CFRP to bend or fail when subjected to external loads or impacts. In the case of static three-point bending, using the conductive properties of the carbon fiber inside the CFRP, the overall damage detection and failure prediction can be carried out by electromagnetic methods. The eddy current coil is used to realize real-time monitoring of damage, and the measured voltage value can be mapped to obtain the load of the sample. This paper conducts theoretical analysis and experimental verification, and obtains the relationship between CFRP stress damage and spatial conductivity change, and proposes a CFRP electromechanical coupling model under quasistatic three-point bending. Combined with the theory of electrically ineffective length, the CFRP three-point bending electromechanical coupling model was revised. Experimental results prove that the revised model can describe the load-conductivity change trend of three-dimensional braided CFRP more accurately, which provides a theoretical basis for monitoring the structural health of CFRP through electromagnetic methods.
\end{abstract}

Keywords: electromagnetic testing; electromagnetic eddy current; three-dimensional angle interlocking woven structure of CFRP

\section{Introduction}

Currently, carbon fiber reinforced polymer materials (CFRP) have been widely used in industrial fields, such as aerospace, military manufacturing, and transportation [1]. After years of development, CFRP has gradually become the main load-bearing structural material in the abovementioned fields. The application of CFRP not only maintains strength and rigidity but also ensures overall safety and prolongs the service life of structural parts. In engineering applications, composite carbon fibers mainly bear loads and resist material deformation and damage caused by external forces. Carbon fibers are prone to matrix cracking, fiber fracture, and delamination [2], which degrades the overall structural mechanical properties.

With the wide application of CFRPs in aerospace, industrial, civil life, etc., composite materials show less damage during use, which is difficult to see with the naked eye. To ensure safety and stability during service, nondestructive testing of CFRPs has attracted wide attention [3], and nondestructive testing technology has been used to detect hidden potential damage without damaging the structure itself. Common detection technologies include ultrasonic detection, radiographic detection, laser ultrasound, and infrared thermal wave detection [4-7], but these technologies usually require couplants, and the detection equipment is relatively large, which is not suitable for fast scanning.

Most nondestructive testing techniques are usually applied to the study of static damage, while the methods mentioned above for the study of dynamic damage of composite 
materials are not very suitable because of the influence of the volume of the testing equipment and environmental conditions. Taking advantage of the good conductivity of carbon fiber materials in CFRP [8], the dynamic damage changes of the internal structure can be mapped through changes in its electrical properties [9-11]. Taking advantage of the good electrical conductivity of carbon fiber, it can be used as a sensor [12] for detection by electrical methods, which obtains voltage signals by injecting current excitation into the measured area for noninvasive imaging [13]. However, the use of electrical impedance testing to study composite materials and the anisotropy and inhomogeneity of laminates still poses great challenges to testing research. Through electromagnetic eddy current nondestructive testing technology, using the principle of electromagnetic induction, the defects in the prefabrication stage of CFRP and the laying sequence can be noncontact detected [14,15], and the damage during service can also be measured [3]. Benjamin Haefner et al. proposed an eddy current sensor array to measure CFRP materials [16]. Miguel A. et al. designed a planar eddy current array probe, which can perform high-speed nondestructive testing for unidirectional CFRP under harsh conditions [17]. Megali G. et al. used COMSOL Multiphysics software based on the A- $\varphi$ method to perform electromagnetic eddy current simulation analysis. The model unit was divided into tetrahedral mesh elements, and the CFRP plate was equivalent to a continuous anisotropic material [18]. When using eddy current coils to study the damage of CFRP, the establishment of its electromechanical coupling model becomes very important.

Many researchers have performed in-depth research on the electromechanical coupling model of carbon fiber bending. For example, Luan [12] and others used carbon fiber as a sensor and embedded it into a three-dimensional (3D)-printed thermoplastic polymer beam. The resistance change of the carbon fiber with the load applied at different positions was explored, and the electromechanical coupling model of the carbon fiber due to the bending deformation was obtained. However, when the embedded carbon fiber is used as a sensor to study its resistance change, the fiber cannot be broken, and when the fiber polymer (FRP) is bent, not only does the resistance change due to bending deformation but the fiber is still connected after damage and breakage, which also causes the resistance to change. For fiber polymers (FRPs), the expansion of the overall damage is usually caused by the fracture of the fiber clusters. Understanding the changes in the FRP microscopic fiber clusters becomes very important for establishing an overall electromechanical model. The fibers in the fiber cluster do not all have the same strength. In contrast, the strength of these fibers usually follows the Weibull distribution [19]. Park et al. introduced the Weibull distribution of fiber strength into the study of resistivity changes under CFRP tensile experiments and proposed the concept of an electrically ineffective length [20].

The abovementioned researchers used the resistance method mainly to study CFRP and obtained the resistance change model with the influence of external factors. In this paper, through theoretical analysis, numerical fitting, and experimental verification, the relationship between CFRP stress damage, and spatial conductivity change is obtained. Combine the "load bending-conductivity" (LBC) model with the "fiber break-conductivity" (FBC) model proposed by Park with reference to the electrically ineffective length, and extend the electromechanical coupling model under three-point bending. Through the mechanical simulation of 3DAWF of different structures, the mechanical simulation results are brought into the model, and the theoretical overall change trend is obtained, and a quasi-static bending test is performed. Two different structures of the specimens were tested by bending, and a full cycle of monitoring by eddy current coil. In the process of being subjected to static load, the electrical properties of CFRP are affected by the two mechanisms of deformation and micro-fiber fracture. In order to accurately predict the load of the sample, this paper proposes an FBC electromechanical coupling model of CFRP under three-point bending. The FBC model, combined with the principle of electrical ineffective length, effectively describes the physical process of fiber fracture and reconnection, and can more accurately predict the load-conductivity change trend of CFRP. In order to verify the reliability of the FBC model, this paper uses eddy current sensor 
real-time in situ monitoring and conducts a dynamic mechanical damage experiment of $3 \mathrm{DAWF}$ in quasi-static three-point bending. The experimental results prove that the FBC model has higher accuracy than the LBC model. We expect to be able to monitor the changes in the material state in real-time. Under the condition that the structural parts are known to bear the maximum load, the conductivity model is combined with the eddy current detection technology to perform full-cycle online health monitoring of the sample structure to realize the prediction of the remaining life of the structural parts.

\section{Conductivity Change Model of CFRP under Three-Point Bending}

Under the condition of quasi-static three-point bending, the CFRP material first undergoes bending deformation and interlayer cracks, and then the internal fibers break sequentially from bottom to top until the fiber completely breaks and fails. In the whole process, the conductivity change of CFRP is composed of two parts: 1 . Macroscopically, the conductivity change of bending strain is caused by bearing load $\sigma_{s t r}$. 2. Microscopically, after the fibers are broken, the conductive path is formed again, resulting in the conductivity change $\sigma_{e f f}$. Therefore, the electrical conductivity $\sigma_{t}$ at time $t$ using the mathematical expression is:

$$
\sigma_{t}=\sigma_{s t r}+\sigma_{e f f}
$$

a. Load bending-conductivity $\sigma_{\text {str }}$ ( $L B C$ model)

The piezoresistance change of the carbon fiber composite material under continuous stress is, the relevant parameters are shown in Figure 1a,b:

$$
\frac{\Delta R_{s t r}}{R_{0}}=k \varepsilon=k \frac{\Delta l}{l}=\frac{k}{l} \int_{0}^{l} \varepsilon(x) d x
$$

where $\Delta R_{s t r}$ is the change in resistance due to strain, $R_{0}$ is the initial resistance, and $k$ is the strain sensitivity, where $\varepsilon(x)$ represents the distribution of strain along the fiber, which depends on boundary conditions and loading methods.

Normal stress $F$ usually satisfies the following three mechanical relations:

$$
\begin{gathered}
N=\int_{A} F d A=0 \\
M_{y}=\int_{A} z F d A=0 \\
M_{z}=\int_{A} y F d A=M=\frac{P l}{4}
\end{gathered}
$$

$N$ is the axial force on the cross section, $M_{y}$ is the coupling moment on the $y$-axis, and $M_{z}$ is the coupling moment on the $z$-axis. 


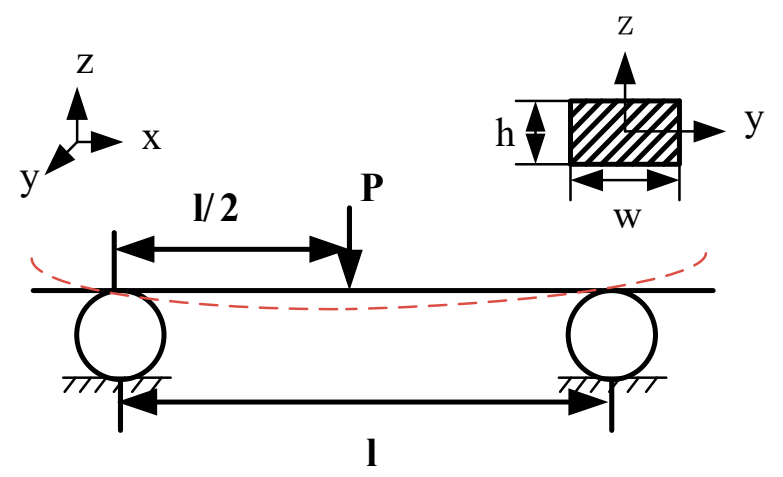

(a)

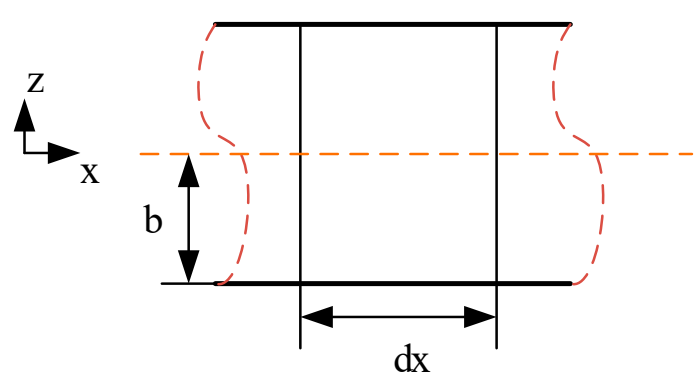

(b)

Figure 1. (a) Related parameters of the applied load (a load is applied to the middle of the sample, and the red dotted line indicates the sample with both ends warped after bending. $h, \omega$, respectively, represent the thickness and width of the sample); (b) sample microsegment ( $b$ is the distance of the carbon fiber layer from the neutral layer, $d x$ is a microsegment of the beam).

Assuming that there is no extrusion between the layers parallel to the neutral layer, combined with the geometric relationship before and after deformation, the physical relationship satisfies Hooke's law:

$$
F=E \varepsilon=E \frac{b}{r}
$$

where $E$ is the modulus of elasticity, $b$ is the distance between the fiber bottom layer and the neutral layer, and $r$ is the radius of curvature of the neutral axis after deformation.

From (3) and (4), the strain in the middle of the template can be obtained as:

$$
\varepsilon=\frac{F}{E}=\frac{M b}{E \int_{A} y^{2} d A}
$$

Combining (2) and (5) can get:

$$
\frac{\Delta R_{s t r}}{R_{0}}=\frac{k}{l} \int_{0}^{\frac{l}{2}} \varepsilon(x) d x=\frac{k}{l} \int_{0}^{\frac{l}{2}} \frac{P x b}{E l \int_{A} y^{2} d A} x d x=\frac{3 k b P l}{2 E h \omega^{2}}
$$

Equation (6) establishes the relationship between the resistance change rate $\frac{\Delta R_{s t r}}{R_{0}}$ and the external load $P$, the loading position, and the distance $b$ between the fiber bottom layer and the neutral layer. Usually, $E, k, l, h, \omega$, and are constant values in the elastic zone. Therefore, the resistance change rate changes with the size of the load. Introducing a sensitivity coefficient $K_{x}$, the equation can be simplified as:

$$
\frac{\Delta R_{s t r}}{R_{0}}=K_{x} P
$$

The sensitivity coefficient $K_{x}$ is constant when the position of the applied load is determined. In this paper, the position where the load is applied is always at $1 / 2$, the sensitivity coefficient is constant, and the impedance change can be monitored in real-time under different load sizes, and Equation (7) shows that as the load increases, the rate of change of resistance also increases, and the two are in a linear relationship.

For carbon fiber composite materials, the volume fraction of internal conductive carbon fibers and the size of the material need to be considered:

$$
R_{0}\left(L_{x}\right)=\frac{L_{x}}{L_{y} e_{0}} \frac{1}{\sigma_{0}} \frac{1}{V_{f}}
$$




$$
R_{t}\left(L_{x}\right)=\frac{L_{x}}{L_{y} e_{0}} \frac{1}{\sigma_{t}} \frac{1}{V_{f}}
$$

From Equations (8) and (9), the initial resistance and the resistance $R_{0}$ and $R_{t}$ at time $t$ can be obtained, where $L_{x}, L_{y}$ is the length of the fiberboard along the $x, y$ axis, $e_{0}$ is the thickness of the fiberboard, $\sigma_{t}, \sigma_{0}$ are the electrical conductivity and initial electrical conductivity along the direction at time $t$, respectively, and $V_{f}$ is the volume fraction of the fiber. Bring these terms into Equation (7) to get:

$$
\begin{gathered}
\frac{\Delta R_{s t r}}{R_{0}}=\frac{R_{t}-R_{0}}{R_{0}}=K_{x} P \\
\sigma_{s t r}=\frac{1}{\left(K_{x} P+1\right)} \sigma_{0}
\end{gathered}
$$

Thus, Equation (10) can be obtained at time $t$. When an external load $P$ is applied, the relationship between the conductivity value and the load size, that is, $\sigma_{t}$ and $P$, is inversely proportional. As the load increases, the conductivity decreases.

\section{b. Fiber break-conductivity $\sigma_{\text {eff }}$ (FBC model)}

The mechanical structure of CFRP can be regarded as a connection of multiple tiny springs, and its electrical structure can be regarded as a series-parallel network of multiple small resistances. As shown in Figure 2b,c, the left half is the schematic model of the micro spring of the mechanical fiber, and the right half is the electrical resistance change model. with the application of load, the mechanical structure of CFRP changes after the carbon fiber breaks. The load-bearing capacity is reduced, its electrical structures are connected to each other due to the broken fibers, and a new conductive network is formed again.

The conductivity of the broken fiber can be derived from the rate of change of the resistance of the broken fiber, which is similar to the derivation process of Equation (10)

$$
\begin{aligned}
\frac{\Delta R_{e f f}}{R_{0}} & =\frac{R_{e f f}-R_{0}}{R_{0}}=\frac{\frac{1}{\sigma_{e f f}}-\frac{1}{\sigma_{0}}}{\frac{1}{\sigma_{0}}} \\
\sigma_{e f f} & =\sigma_{0} /\left(\frac{\Delta R_{e f f}}{R_{0}}+1\right)
\end{aligned}
$$

Based on the concept of electrically ineffective length proposed by Park, a model was proposed to predict the change in electrical resistance due to sample degradation and fiber breakage. Therefore, we added the electrical conductivity change caused by fiber damage to the overall electrical conductivity model. In the series/parallel array model, the overall failure resistance change rate can be expressed as:

$$
\frac{\Delta R_{e f f}}{R_{0}}=\frac{(1+k \varepsilon)}{\exp \left[-\left(\frac{l_{e c}}{L_{x}}\right)\left(\frac{E_{f} \varepsilon}{\chi}\right)^{m}\right]}-1
$$

$l_{e c}$ is the electrically ineffective length; that is, due to the fracture and damage of the carbon fiber, the original conductive path can no longer carry current, and the adjacent contact fiber is a new conductive path, so the electrically ineffective length is the distance between adjacent contact points, as shown in Figure 2d. As shown, the value of $l_{e c}$ is the average length of the fiber contact point, $E_{f}$ is the overall Young's modulus, and $\chi, m$ are the material failure parameters of the Weibull distribution. 


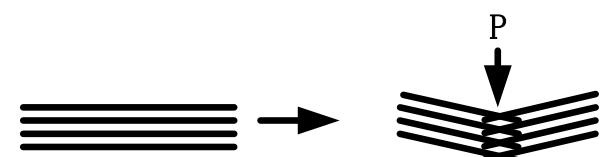

(a)
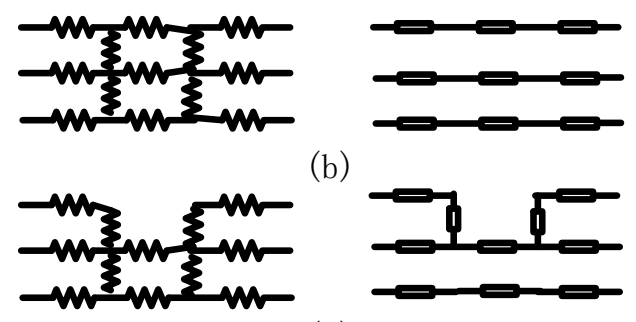

(c)
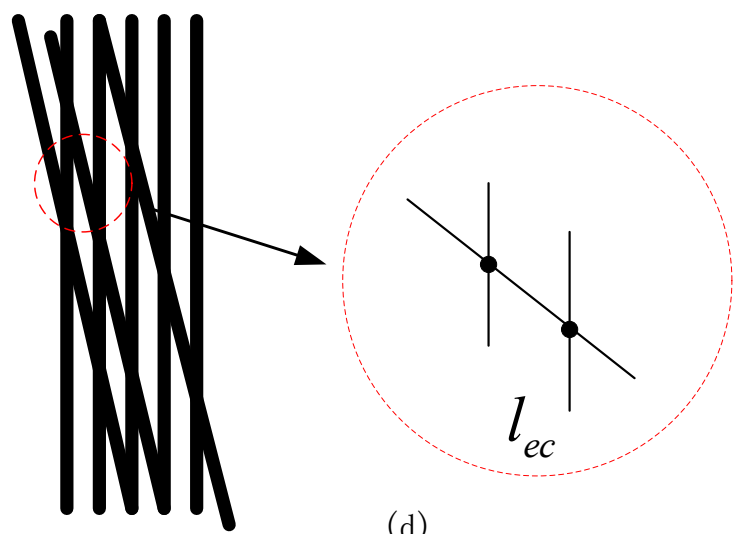

(d)

Figure 2. Two-dimensional micrograph of CFRP (a) CFRP under load and bending fracture; (b) electrical model of CFRP in good condition; (c) electrical model of damaged CFRP (The left half is the schematic model of the micro spring, and the right half is the resistance change model); and (d) schematic diagram of the electrically inactive length $l_{e c}$ of carbon fiber originating from the contact between the fibers.

Therefore, the overall conductivity change is affected by the bending strain of the specimen and the fiber fracture. Combining Equations (11) and (12) to modify the previous LBC model (10), we obtain:

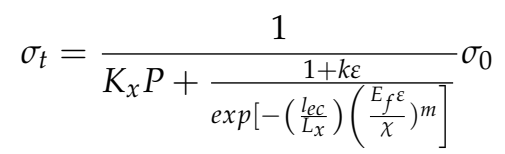

From Equation (13), as the load $P$ increases, the electrical conductivity decreases. From a macroscopic point of view, the bending of the sample leads to a change in the electrical conductivity of the overall resistance. From a microscopic point of view, the fracture of internal fibers rebuilds new conductive paths and will continue to affect the electrical properties of CFRP. The bending strain will be the main influencing factor for changes in electrical conductivity. This model is applicable to all CFRP samples.

\section{Experimental Materials}

The experimental sample uses two three-dimensional angle interlocking woven carbon fiber composite materials (3DAWFs), a three-dimensional weave (PTW) structure, and a twill three-dimensional weave structure (TTW), and the carbon fiber is T800-12K. To damage the outer surface of the sample only by bending, according to the sample preparation guide D5687/D5687M, the standard span-thickness ratio of the sample is 32:1, the thickness of the standard sample is $3.8 \mathrm{~mm}$, and the width is $13 \mathrm{~mm}$. The length of the specimen is $20 \%$ longer than the supporting span. The span of the bending machine support roller is $121.6 \mathrm{~mm}$, and the sample length is approximately $146 \mathrm{~mm}$. The overall layup is 4 layers.

During the layup process, 5284 high-temperature epoxy resin is inserted between the prepregs to control the internal contact and fiber volume fraction of the CFRP laminate. The carbon fiber content is $55 \pm 3 \%$. Standard ASTMD7264 was used to test the three-point bending performance of the two structural layer interwoven composite materials.

Both structural lining warp layers on-line woven fabrics are composed of three sets of system yarns. The stuffer warp yarn (red) is stretched along the fabric forming direction. The layers are connected along the forming direction, and the yarns are sinusoidal in the interior as knotted warps (blue). The weft yarn (yellow) perpendicular to the fabric-forming direction is generally straightened inside the fabric.

Compared with TTW three-dimensional materials, the knotted warp yarn of PTW passes through two weft yarns in one cycle, and TTW three-dimensional woven material 
passes through four warp yarns in one cycle, as shown in Figure 3a,b. The two materials connect the two layers of carbon fiber woven fabric through the knotted warp yarn, thereby obtaining better high delamination resistance and high strength in the thickness direction. The relevant parameters of carbon fiber are shown in Table 1.

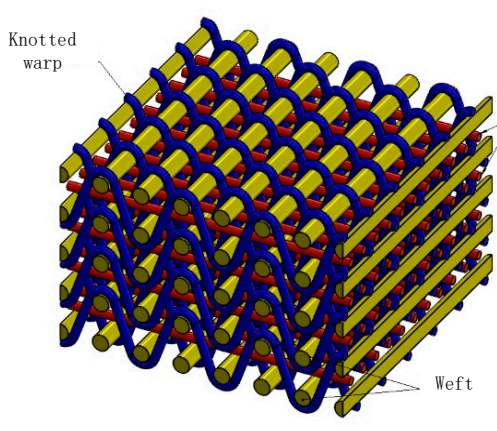

(a)

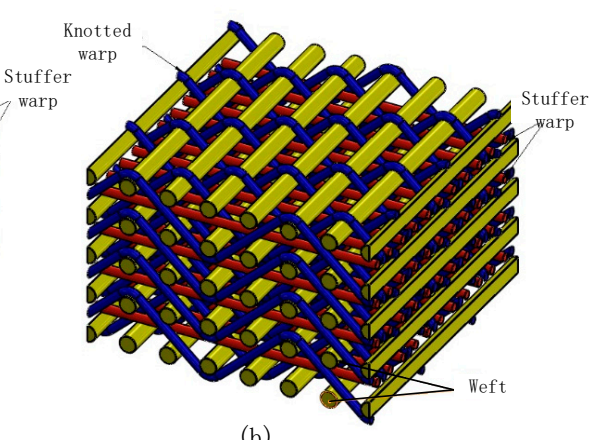

(b)

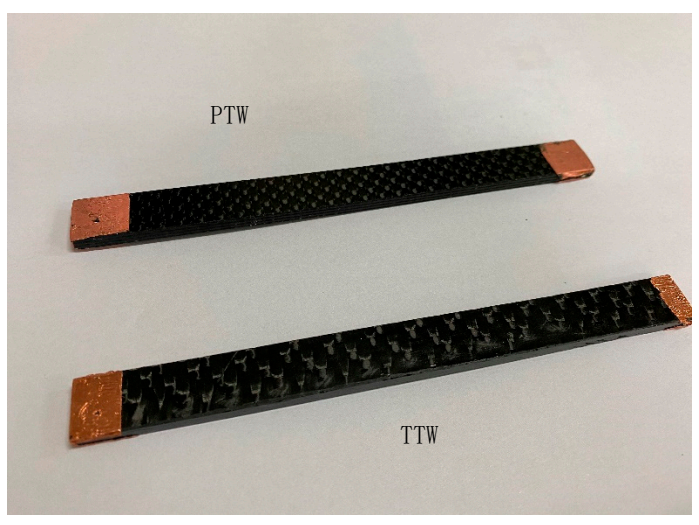

(c)

Figure 3. (a) Plain weave structure (PTW), (b) twill weave structure (TTW), and (c) sample physical picture.

Table 1. Carbon fiber performance parameters.

\begin{tabular}{cccccc}
\hline Model & $\begin{array}{c}\text { Density } \\
\left(\mathbf{g} / \mathbf{c m}^{\mathbf{3}} \mathbf{)}\right.\end{array}$ & $\begin{array}{c}\text { Tensile Strength } \\
\mathbf{( M P a )}\end{array}$ & $\begin{array}{c}\text { Elongation at Break } \\
\mathbf{( \% )}\end{array}$ & $\begin{array}{c}\text { Modulus of Elasticity } \\
\mathbf{( G P a )}\end{array}$ & $\begin{array}{c}\text { General Rate } \\
\mathbf{( \% )}\end{array}$ \\
\hline $\mathrm{T} 800-12 \mathrm{~K}$ & 1.79 & 5678 & 2.32 & 290 & 1.5 \\
\hline
\end{tabular}

The change in CFRP resistance is greatly affected by the size, and the conductivity is between $5 \times 10^{3} \sim 5 \times 10^{4} \mathrm{~S} / \mathrm{m}$. Therefore, to accurately measure the resistance of the sample, it is necessary to avoid and reduce contact resistance as much as possible. We polished the upper and lower surfaces of the sample along the width and thickness directions, removed the surface resin to expose the fibers, and then applied conductive silver glue to the surface evenly. After static air-drying, conductive copper tape was used to stick to the surface of the silver glue to increase the contact area between the positive and negative chucks of the impedance analyzer and the sample, thereby reducing the contact resistance, as shown in Figure 3c.

\section{Simulation}

According to the difference between the structure of plain weave and twill weave, modeling with COMSOL Multiphysics finite element analysis software, in the modeling, the state of binding warp yarn is simulated by sweeping a parameterized curve, and the warp yarn and weft yarn are arranged in an array. Consistent size and related parameters, 
reflecting only the CFRP structure caused by differences in the weave samples, are necessary for simulation.

\subsection{Three-Point Bending Solid Mechanics Part}

To explore the situation of the two structures under a three-point bending force, the length of the middle part of the model is $3 \mathrm{~mm}$ to simulate the width of the lower indenter, and two cylinders with a span of $122 \mathrm{~mm}$ are placed under the sample as fixed constraints to simulate the supporting roller. Set the downward force on the upper surface of the middle part to simulate the applied load P. PTW and TTW bending deflection curves under force as shown in Figure 4. The relevant mechanical setting parameters are shown in Table 2.

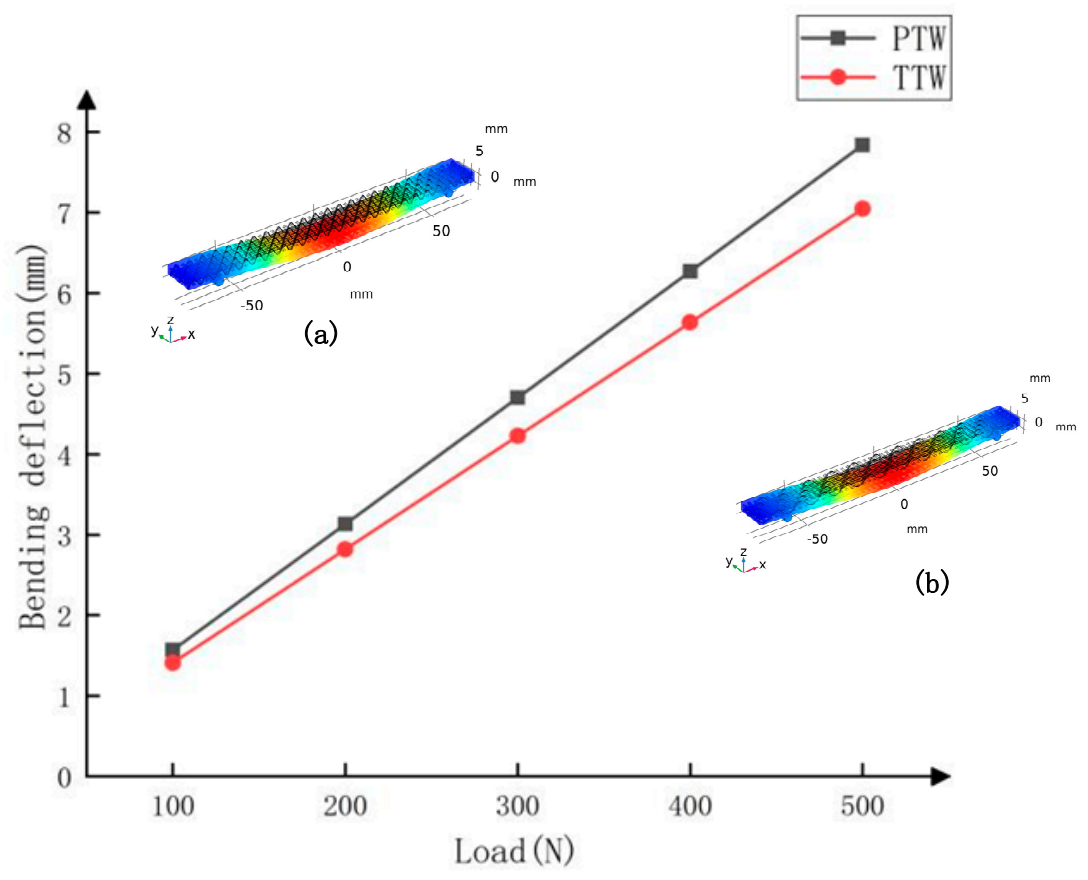

Figure 4. PTW and TTW bending deflection curves under force; cloud diagram of displacement changes after bending under force. (a) PTW, and (b) TTW.

Table 2. Mechanical simulation parameters.

\begin{tabular}{ccc}
\hline & Carbon Fiber & Epoxy Resin \\
\hline Density $\left(\mathrm{kg} / \mathrm{m}^{3}\right)$ & 1790 & 1100 \\
Poisson's ratio & 0.307 & 0.35 \\
Elastic modulus $(\mathrm{GPa})$ & 290 & 4 \\
\hline
\end{tabular}

The load P on the PTW and TTW three-dimensional braided structure is increased from $100 \mathrm{~N}$ to $500 \mathrm{~N}$ to obtain the load-strain curve. From Figure 5 of the force bending deflection curve, as the load increases, the deflection growth rate of the PTW sample is greater than the deflection growth rate of the TTW structure sample. Under the same stress, the TTW structure should change less. 


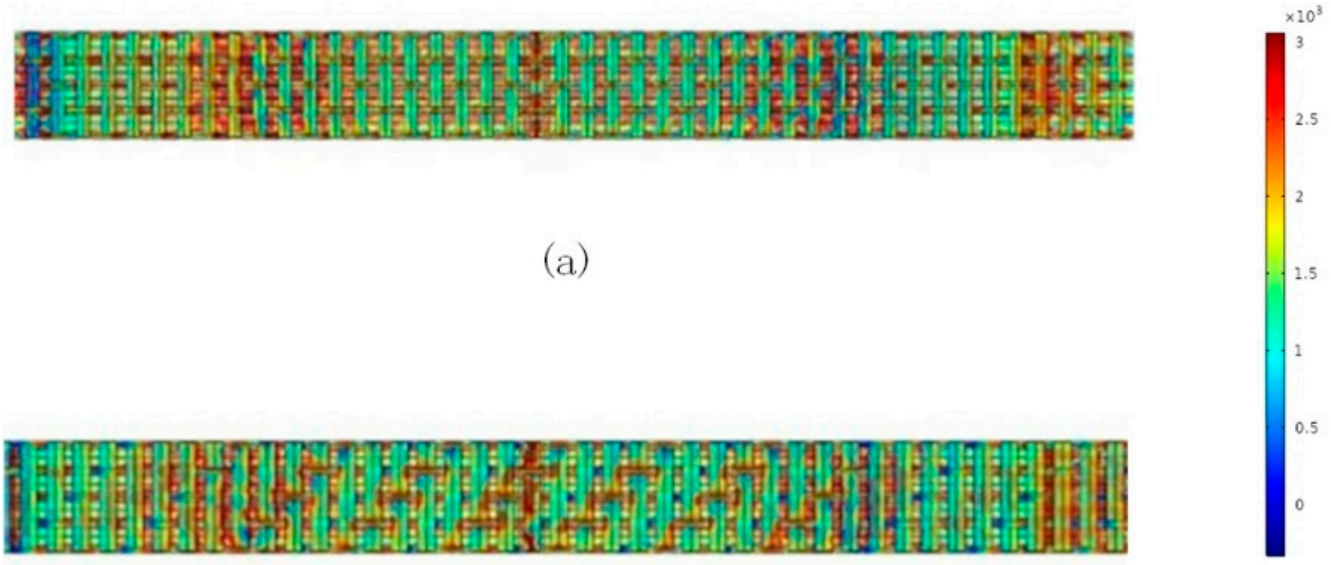

(b)

Figure 5. When the same load of $500 \mathrm{~N}$ is applied, the component distribution diagram of the principal stress in the $z$-axis direction (MPa): (a) the top view of the PTW structure, and (b) the top view of the TTW structure.

For the PTW structure, the top view of the stress distribution shows that along the length of the template, the support roller and the stress area are the reddest. In the middle position between the support rollers, the red color is concentrated on the knotted warp yarn and the stuffer warp yarn. In the areas on both sides of the support roller, the red color is concentrated on the knotted warp yarn, indicating that the knotted warp and the stuffer warp mainly bear most of the stress in the $z$-axis direction. For the top view of the stress distribution of the TTW structure, the dark red part appears as an oblique stripe, indicating that the knotted warp yarn bears most of the stress in the $z$-axis direction.

The similarity between the two structures is that the warp yarns bear greater stress. Because the knotted warp yarns are laid into the weft yarn system at a certain buckling change angle along the thickness direction, they present a wavy state. The buckling and fluctuating knotted warp yarns effectively transfer the bending load to other areas in the thickness direction, while the weft yarns are distributed flatly in the yarn system, and there is no special stress distribution area. The difference is that the twill weave structure can withstand a higher level of stress load than the plain weave structure because of its high fabric density, and the knotted warp yarns bear most of the stress.

\subsection{Electromagnetic Simulation}

A dual-coil sensor is designed as shown in Figure 6. A small coil is placed in the large coil, where the large coil is the excitation coil and the small coil is the collection coil, and the sensor is placed in the center of the sample. In the simulation, the two types of structures choose to use the LBC model to solve the conductivity value within the average load pressure range. 


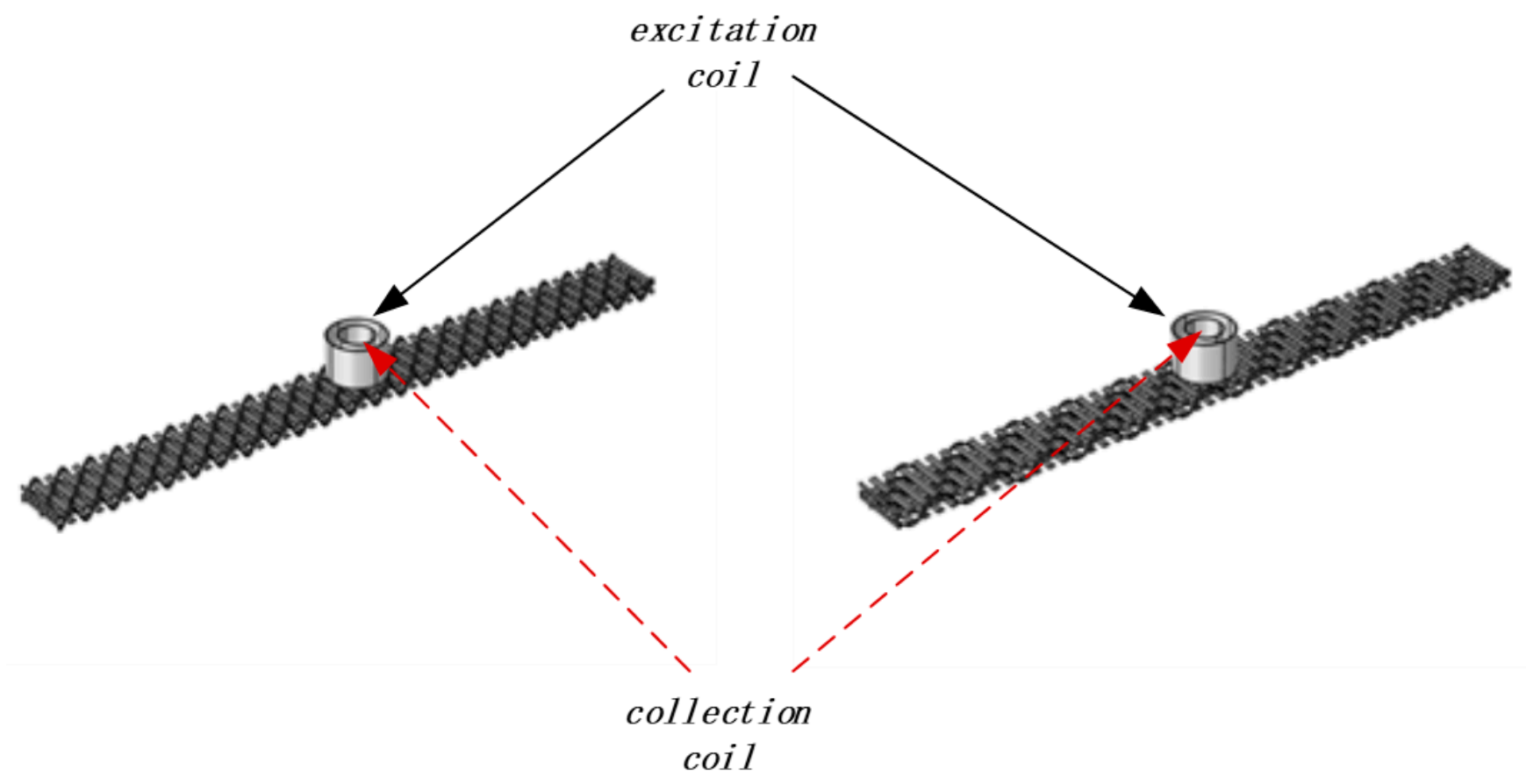

Figure 6. Electromagnetic coil detection model, the large coil is the excitation coil and the small coil is the collection coil.

The lift-off height of the dual-coil sensor is $0.3 \mathrm{~mm}$ from the sample surface, and the coil current is set to $1 \mathrm{~A}$. To make the impedance of the sample appear inductive and allow the current to pass through the sample better, the frequency is set to a high frequency of $1 \mathrm{MHz}$ in the simulation.

The height of the two coils is $8.6 \mathrm{~mm}$, and the number of turns is $100 \mathrm{r}$. The model is shown in Figure 6. To obtain a strong signal as a whole, the radius of the excitation coil is set to $6.2 \mathrm{~mm}$.

For the selection of the radius of the collection coil, to obtain the size of the collection coil most sensitive to defects, a series of internal diameter values of the collection coil with a radius of $5 \sim 2.8 \mathrm{~mm}$ was tested in the simulation, and coils with different radii were placed in intact and defective tests. In the sample, the voltage difference between the two conditions is measured.

From Figure 7b, a series of coils with different radius are tested, and it can be found that as the coil radius decreases, the overall signal also decreases. However, the difference between the voltage values in the non-damaged and damaged state is found to be the largest when the radius is $4.6 \mathrm{~mm}$, indicating that the radius of $4.6 \mathrm{~mm}$ is the most sensitive to damage response.

When the coil radius is determined and the thickness of the coil is optimized, Figure 7c, it can be found that as the thickness of the coil increases, the overall signal is in a state of decreasing. Similarly, the voltage difference between the non-damaged and damaged state is made. When the thickness is $1 \mathrm{~mm}$, the voltage difference at this time is the largest.

As shown in Figure 7d, when the size of the collection coil is $4.6 \mathrm{~mm}$, the voltage value changes most obviously, which is better than the other 6 groups of coil sizes, and the sensitivity is $46 \%$ higher than the average.

Simultaneously, the thickness of the coil was simulated. The diameter of the coil copper wire was $0.2 \mathrm{~mm}$, so it increased from $0.2 \sim 1.2 \mathrm{~mm}$ with a step length of $0.2 \mathrm{~mm}$. As shown in Figure 7e, when the coil was $1 \mathrm{~mm}$ thick, the sensitivity to damage was the best compared to the overall average increase of $278 \%$. 


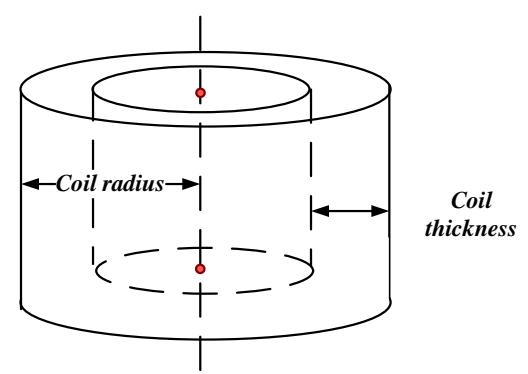

(a)

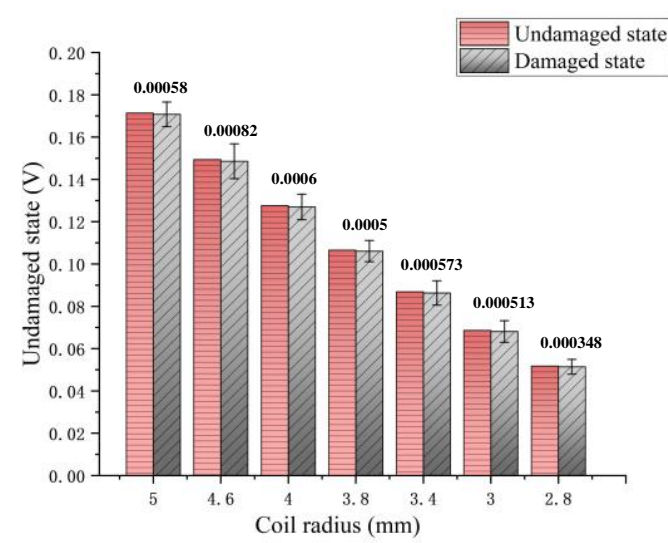

(b)

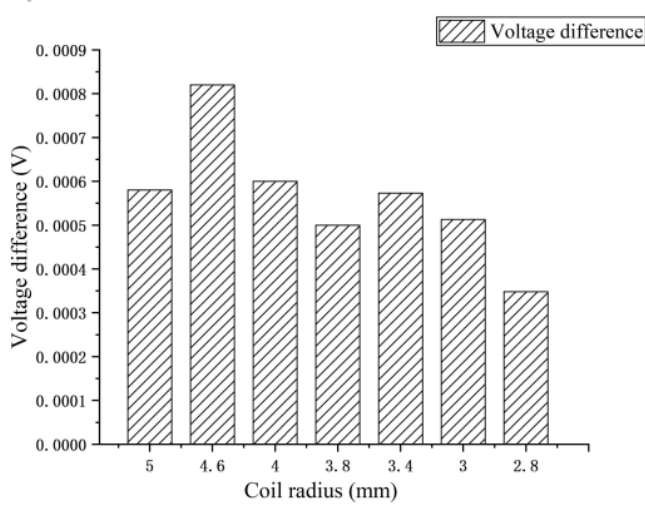

(d)

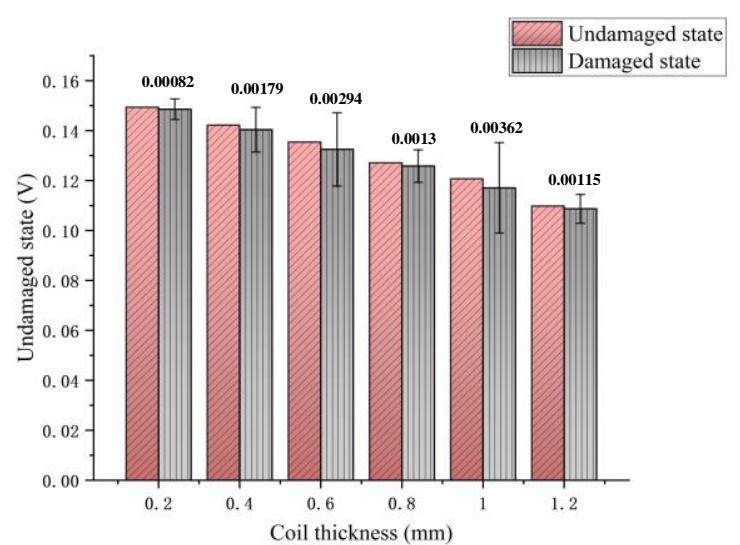

(c)

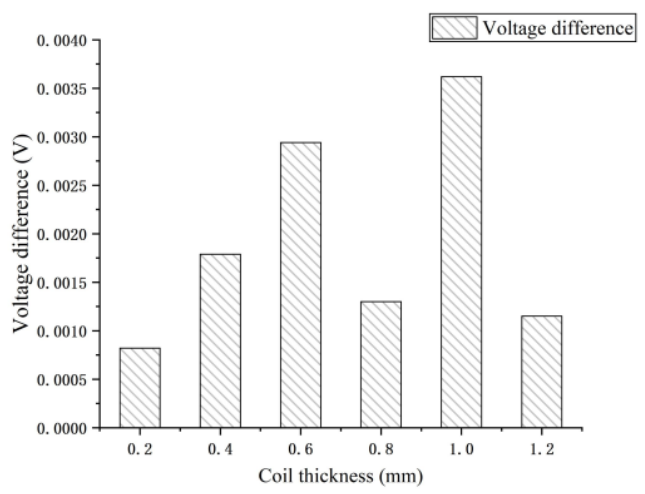

(e)

Figure 7. Coil size sensitivity test in simulation: (a) Schematic diagram of coil optimization parameters; (b) collecting coil radius optimization test; (c) coil thickness optimization test; (d) histogram of voltage difference with the change of coil radius; and (e) histogram of voltage difference as the thickness of the coil changes.

In the simulation, we use the LBC and FBC models to determine the conductivity. We choose a 500-N load and observe the trends of the two models, as shown in Figure 8. The results show that the FBC model is used to solve the conductivity. The parameters are shown in Table 3. The change trend is greater than the change trend of the LBC model, but the difference between the two is not large, showing that the damage caused by the fiber load will affect the electrical conductivity. 


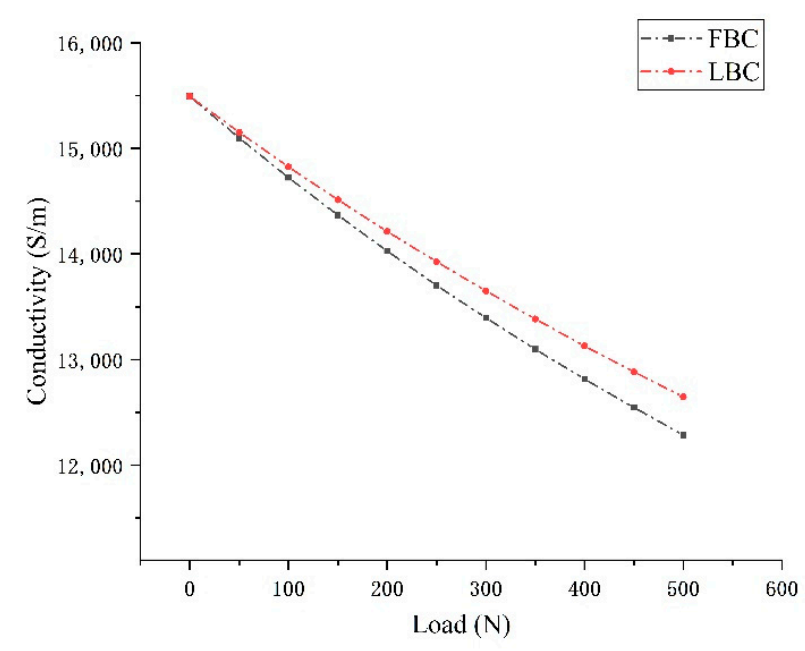

Figure 8. Comparison of the LBC and FBC conductivity models (Load = 500 N).

Table 3. Related parameters of the electromagnetic simulation.

\begin{tabular}{cc}
\hline Coil & Parameter \\
\hline Excitation coil radius & $6.2 \mathrm{~mm}$ \\
Collection coil radius & $4.6 \mathrm{~mm}$ \\
Number of turns & $100 \mathrm{r}$ \\
Lift-off height & $0.3 \mathrm{~mm}$ \\
current/frequency & $1 \mathrm{~A} / 1 \mathrm{MHz}$ \\
\hline CFRP & Parameter \\
\hline Initial conductivity of warp & $\sigma_{x}=15,500 \frac{\mathrm{S}}{\mathrm{m}}$ \\
& $\sigma_{y}=50 \mathrm{~S} / \mathrm{m}$ \\
& $\sigma_{z}=10 \mathrm{~S} / \mathrm{m}$ \\
Initial conductivity of weft yarn & $\frac{\sigma_{x}=50 \mathrm{~S}}{\mathrm{~m}}$ \\
& $\frac{\sigma_{y}=15,500 \mathrm{~S}}{\mathrm{~m}}$ \\
& $\sigma_{z}=10 \mathrm{~S} / \mathrm{m}$ \\
\hline
\end{tabular}

The displacement field change of the mechanical simulation is divided into three parts according to the different colors, as shown in the displacement field deflection change cloud diagram in Figure 9a. C1 is the dark red area with the maximum strain part, the length is approximately $30 \mathrm{~mm}$; C2 is the medium strain part from red to light blue, and the total length is approximately $56 \mathrm{~mm}$; and C3 is the dark blue part, which has the lowest strain, and the length is approximately $60 \mathrm{~mm}$.

The conductivity values of the two structures are set within the average load pressure range. The different load sizes of the two structures are the average load that the two structures can withstand in the reference experiment. The PTW is $450 \mathrm{~N}$ and the TTW structure is $670 \mathrm{~N}$. After calculating the LBC model and the FBC model separately, the coil voltage curves of the two structures are obtained, as shown in Figure 9c. The coil voltages of the two structures have the same change trend, and they decrease exponentially with increasing load.

The predicted value of the FBC model is lower than the predicted value of the LBC model, and the overall downward trend is faster, but the difference between the two is not very large, indicating that the bending strain is the main reason for the change in electrical conductivity.

The bottom carbon fiber is cracked due to bending, and then the broken fiber is reconnected. The new conductive path formed has an impact on the overall conductivity change, but it is not the main factor. 


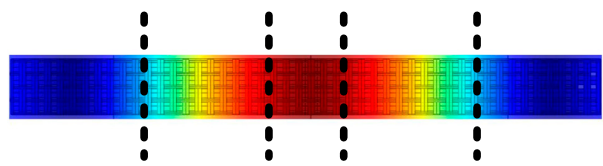

(a)

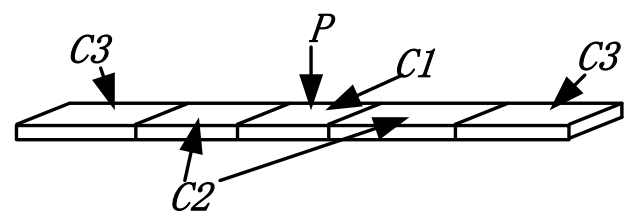

(b)

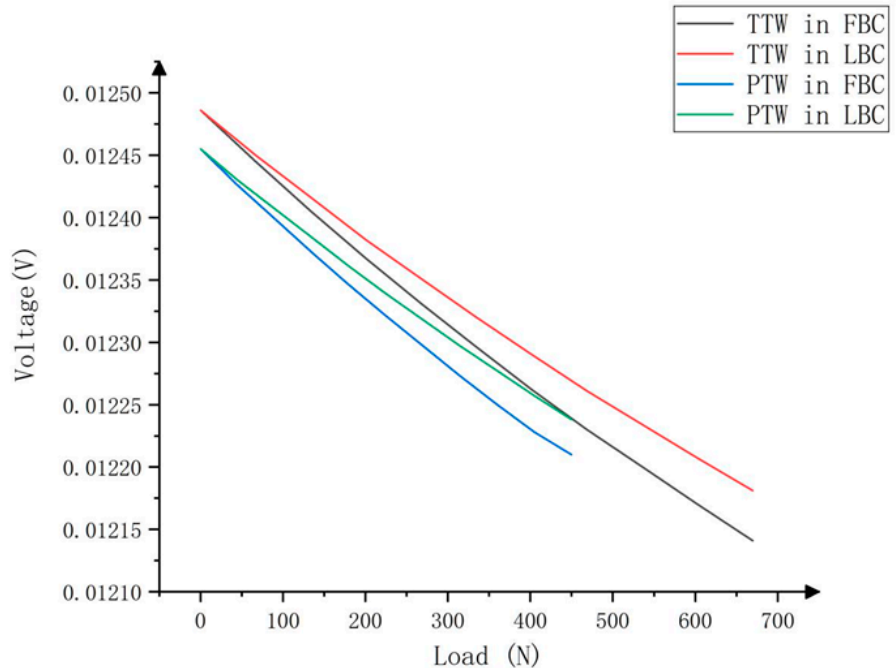

(c)

Figure 9. (a) Strain cloud diagram of the displacement field; (b) schematic diagram of the division of Ci (I = 1, 2, 3); (c) PTW, TTW structure electromagnetic simulation results.

\section{Experiment}

\subsection{Experimental Methods and Equipment}

This section discusses a 3DAWF sample, an electromagnetic eddy current real-time monitoring system, and a universally powerful machine. In the experiment, a single longitudinal load was applied to the carbon fiber sample through a universal strength machine, and a three-point bending experiment was carried out until the sample fractured. During this process, an electromagnetic eddy current real-time monitoring system was used to monitor the sample throughout the whole cycle.

As shown in Figure 10, in the experiment, the sample was placed in the middle of the supporting roller, and both ends were fixed on the supporting roller to prevent the sample position from shifting during the bending process. To obtain the most obvious change trend, the monitoring probe is fixed directly under the applied load of the sample, and the probe and the load nose are in the same straight line. The AG1KNE model universal strength machine produced by the Shimadzu Corporation was used to apply the load at a loading rate of $1 \mathrm{~mm} / \mathrm{min}$, and the load was applied until the sample broke. For real-time monitoring, a DMET multichannel digital electromagnetic tomography online imager is selected to detect the electromagnetic characteristics of the sample.

During the experiment, due to the application of external force, the sample was bent, the fibers were broken, and the original conductive network was destroyed, which caused the conductivity of the sample to change.

Thus, the mapping can be utilized with the dependence of the conductivity of the load to explore the relationship between the load voltage signal and the eddy current coil. 


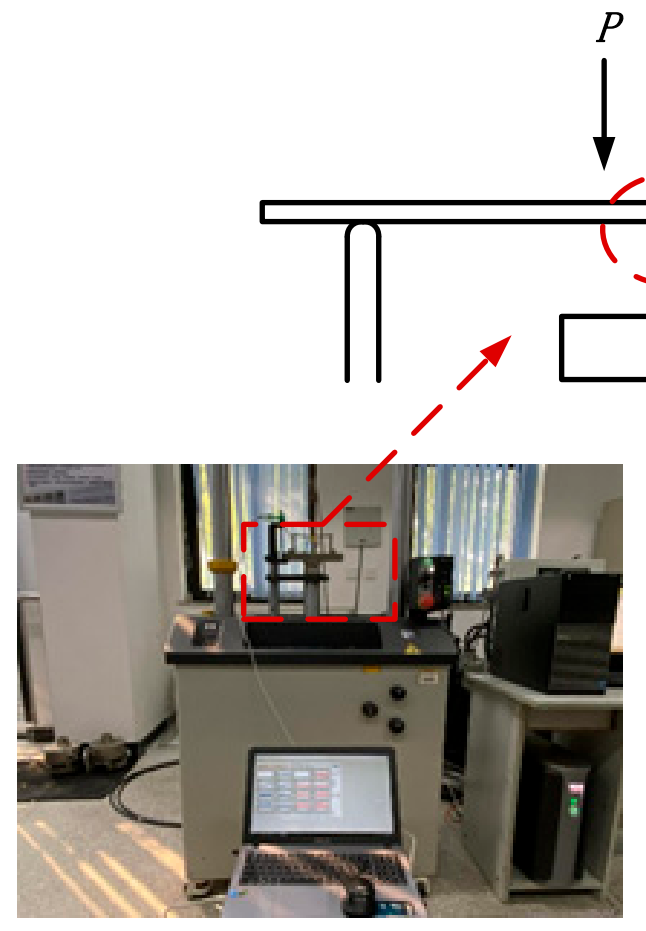

(b)

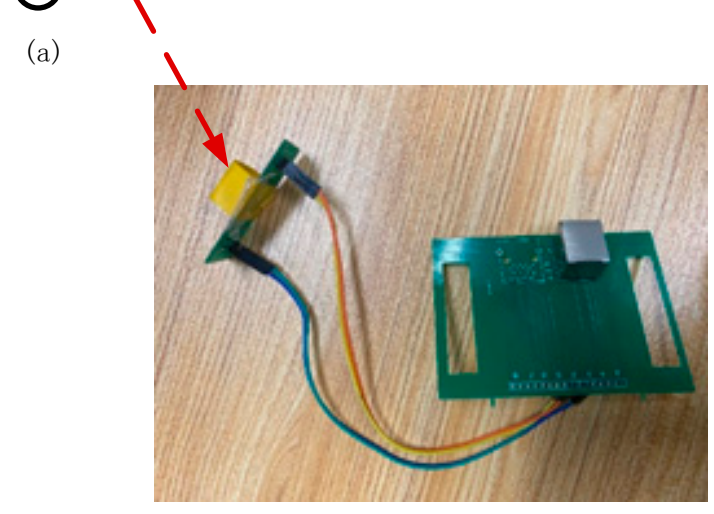

(c)

Figure 10. Quasistatic bending test system and sensor probe (a) is a simple schematic diagram of the experimental system; (b) the actual scene photo; and (c) the eddy current coil sensor.

\subsection{Experimental Results}

As shown in Figure 11, the resistance value of the TTW and PTW weave samples before and after the load is bent is measured using an impedance analyzer to obtain the resistance change rate $\Delta R / R_{0}$, which is divided by the load applied to it to obtain the $K_{x}$. After calculation, the value ranges of PTW and TTW are $7.3 \times 10^{-3} \sim 9.1 \times 10^{-3}$ and $8.5 \times 10^{-4} \sim 12.1 \times 10^{-4}$, respectively.

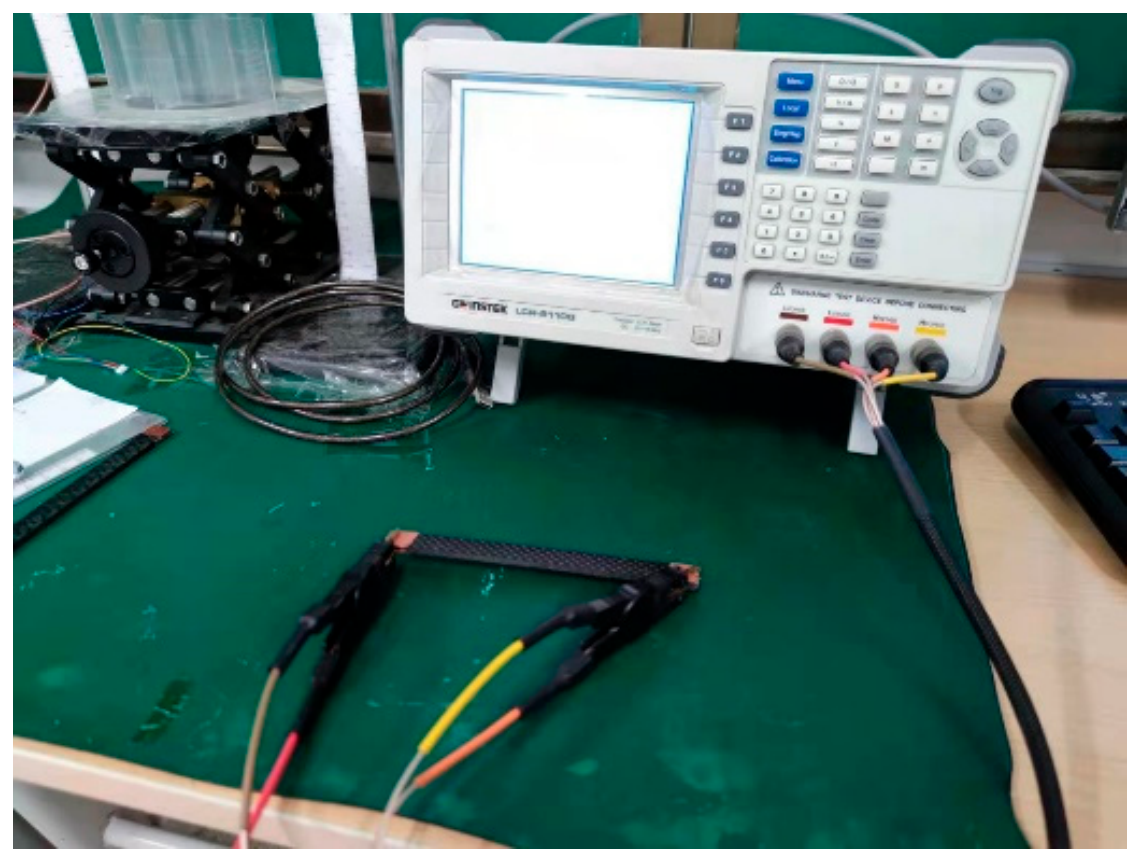

Figure 11. Impedance analyzer measures the rate of change of resistance. 
In the experiment, the constant pressing rate of the universal strength machine is set to $1 \mathrm{~mm} / \mathrm{min}$. To prevent the sample from slipping, a $2 \mathrm{~N}$ preload is applied. Figure 12 shows the deflection-load curve of the two structures. The average loads of the PTW structure and TTW structure samples are $465.98 \mathrm{~N}$ and $671.98 \mathrm{~N}$, respectively. The figure shows that before fracture, the load increases linearly with the deflection. When the deflection reaches $10 \mathrm{~mm}$, the load decreases rapidly. The specimens of the two structures fail. At this time, a large number of fibers break, and the matrix cracks.

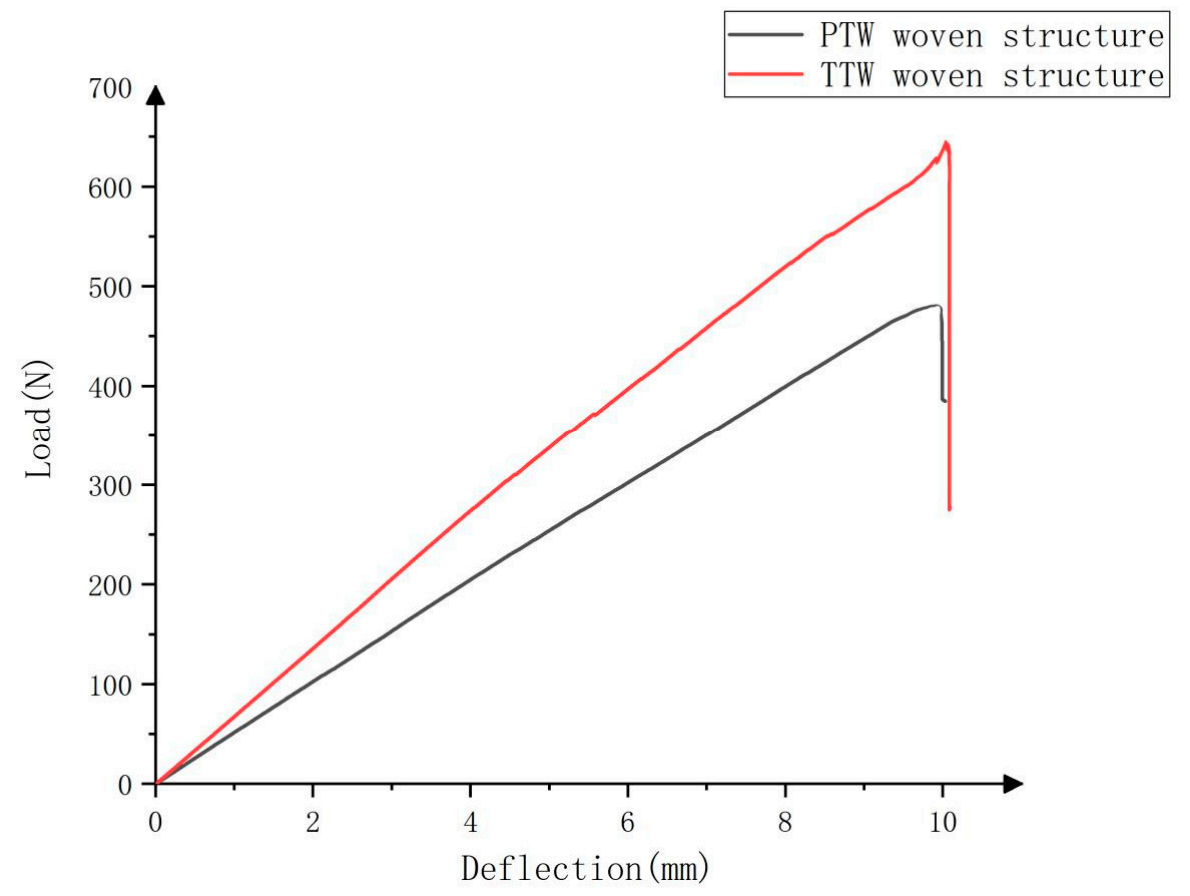

Figure 12. Deflection-load curves of the two structures.

In the experiment, the samples of the two structures were monitored for the whole cycle. To reduce the error, we averaged every 100 data values measured to obtain a new average data value and made a scatter plot of the final multiple average values. The TTW structure can bear greater stress, the measurement time is longer, and the voltage average scatter diagram of the TTW structure is relatively denser than the voltage average scatter diagram of the PTW structure. When the sample breaks, the full cycle data collection stops.

Due to the stretching of the fibers in the early stage and the breakage of the fibers in the later stage and reattachment, the electrical resistance of the material itself continues to increase, but the growth rate decreases, so the electrical conductivity of the material also decreases and tends to be flat. This is because matrix cracking occurs in the early stage itself. The greater the applied load, the smaller the stress or strain ratio, and the faster the damage development. In the later stage, the carbon fiber in the matrix will gradually break with increasing load, which means that the crack density tends to be saturated. The measured average voltage value of the coil changes significantly at the end, and the overall trend decays exponentially.

We normalize the average coil voltage measured in the experiment, use the same coefficient to normalize the coil voltage value obtained in the simulation using the two models of LBC and FBC, and compare the two, as shown in Figure 13. The LBC model has a large gap, but the simulation results of the FBC model fit well with the experimental results. 

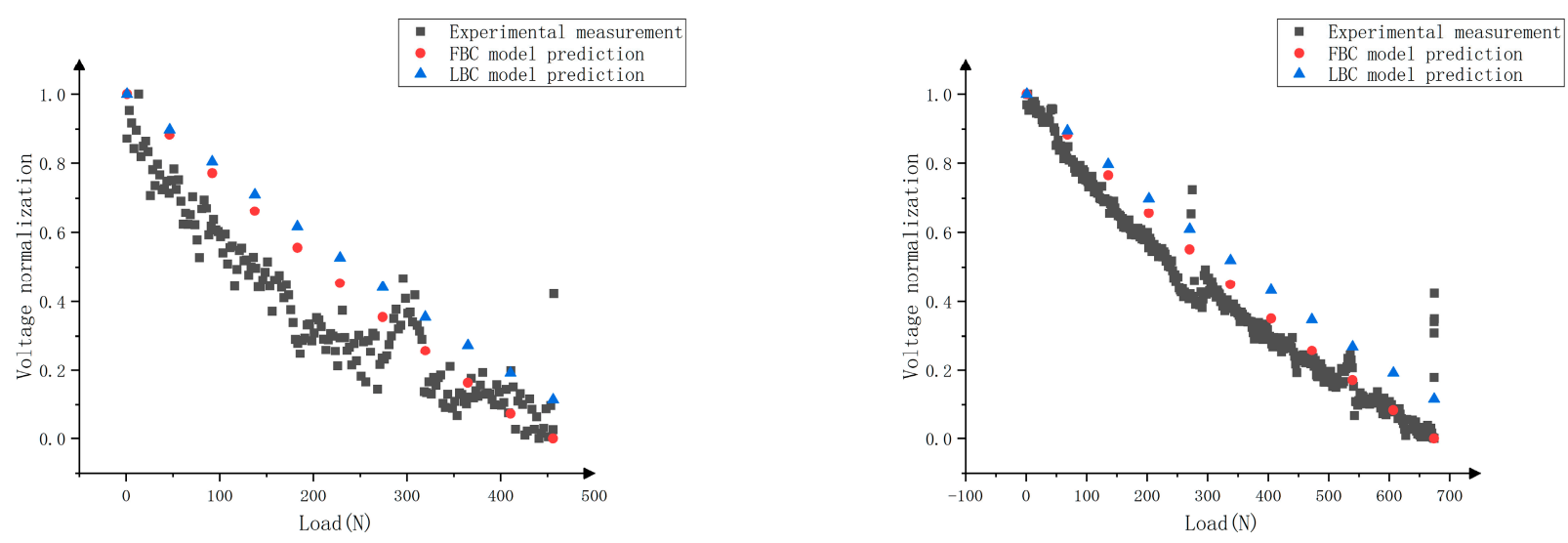

Figure 13. Normalized comparison between the simulation and experimental results. The (left) picture shows the PTW structure, and the (right) picture shows the TTW structure.

In summary, the FBC model is used for prediction. The effect is better, and it can more accurately describe the stress-conductivity change trend of three-dimensional braided CFRP. Therefore, we can use the electromechanical coupling relationship obtained by the FBC model to reflect the physical damage changes during the bending process of the sample.

\section{Conclusions}

1. Due to the unique binding warp yarn embedded in the thickness direction of the threedimensional angle interlocking structure, the material has better high delamination resistance and high strength in the in-plane direction and the thickness direction than the ordinary two-dimensional woven structure material. The buckling and fluctuating binding warp yarns effectively transfer the bending load to other areas in the thickness direction, while the weft yarns are distributed flatly in the yarn system, and there is no special stress distribution area. The difference is that TTW has a high fabric density, and the binding warp yarn bears most of the stress. Therefore, when a quasistatic load is applied, the TTW structure can withstand a higher level of load than the PTW structure when it breaks.

2. When the specimen is bent, matrix cracking occurs first. The greater the applied load, the smaller the stress or strain ratio, and the faster the damage will develop. In the latter half, when the carbon fiber in the matrix gradually breaks with the increase of the load, it means that the crack density tends to be saturated, and at this time, the damage of the material becomes slow. In the bending test, due to changes in the internal fibers, such as stretching and breaking, the electrical resistance of the material itself increases, so the electrical conductivity of the material also decreases accordingly. The electrical conductivity of the material changes so that the magnetic field excited by the excitation coil in the material changes accordingly. Therefore, using the principle of electromagnetic induction, the voltage collected by the fabricated coil probe can indirectly indicate the physical damage change during the bending process of the sample.

3. Combined with the theory of electric ineffective length, the previous three-point bending electromechanical coupling model (LBC) of three-dimensional braided CFRP was revised. Experimental results prove that the revised FBC model can more accurately describe the stress-conductivity change trend of three-dimensional braided CFRP, which provides a theoretical basis for monitoring the structural health of CFRP through electromagnetic methods. 
Author Contributions: Methodology, R.Z.; formal analysis, data curation, L.Z.; writing-original draft preparation, H.F.; Conceptualization, W.Y. All authors have read and agreed to the published version of the manuscript.

Funding: This research was funded by National Natural Science Foundation of China, grant number 62071328 .

Institutional Review Board Statement: The study was conducted according to the guidelines of the Declaration of Helsinki, and approved by the Institutional Review Board.

Data Availability Statement: Not applicable.

Acknowledgments: This work was supported by National Natural Science Foundation of China under Grant 62071328.

Conflicts of Interest: The authors declare no conflict of interest.

\section{References}

1. Cheng, L.; Tian, G.Y. Surface Crack Detection for Carbon Fiber Reinforced Plastic (CFRP) Materials Using Pulsed Eddy Current Thermography. IEEE Sensors J. 2011, 11, 3261-3268. [CrossRef]

2. Wen, J.; Xia, Z.; Choy, F. Damage detection of carbon fiber reinforced polymer composites via electrical resistance measurement. Compos. Part B Eng. 2011, 42, 77-86. [CrossRef]

3. De Goeje, M.; Wapenaar, K. Non-destructive inspection of carbon fibre-reinforced plastics using eddy current methods. Compos. 1992, 23, 147-157. [CrossRef]

4. Wronkowicz-Katunin, A.; Dragan, K.; Lis, K. Assessment of uncertainty in damage evaluation by ultrasonic testing of composite structures. Compos. Struct. 2018, 203, 71-84. [CrossRef]

5. Schilling, P.J.; Karedla, B.R.; Tatiparthi, A.K.; Verges, M.A.; Herrington, P.D. X-ray computed microtomography of internal damage in fiber reinforced polymer matrix composites. Compos. Sci. Technol. 2005, 65, 2071-2078. [CrossRef]

6. Bustamante, L.; Jeyaprakash, N.; Yang, C.-H. Hybrid laser and air-coupled ultrasonic defect detection of aluminium and CFRP plates by means of Lamb mode. Results Phys. 2020, 19, 103438. [CrossRef]

7. Hedayatrasa, S.; Poelman, G.; Segers, J.; VAN Paepegem, W.; Kersemans, M. Performance of frequency and/or phase modulated excitation waveforms for optical infrared thermography of CFRPs through thermal wave radar: A simulation study. Compos. Struct. 2019, 225, 111177. [CrossRef]

8. Nishio, Y.; Todoroki, A.; Mizutani, Y.; Suzuki, Y. Piezoresistive effect of plain-weave CFRP fabric subjected to cyclic loading. Adv. Compos. Mater. 2016, 26, 1-15. [CrossRef]

9. Antonucci, V.; Giordano, M.; Nicolais, L.; Calabrò, A.; Cusano, A.; Cutolo, A.; Inserra, S. Resin flow monitoring in resin film infusion process. J. Mater. Process Technol. 2003, 143-144, 687-692. [CrossRef]

10. Danisman, M.; Tuncol, G.; Kaynar, A.; Sozer, E.M. Monitoring of resin flow in the resin transfer molding (RTM) process using point-voltage sensors. Compos. Sci. Technol. 2007, 67, 367-379. [CrossRef]

11. Matsuzaki, R.; Kobayashi, S.; Todoroki, A.; Mizutani, Y. Full-field monitoring of resin flow using an area-sensor array in a VaRTM process. Compos. Part A Appl. Sci. Manuf. 2011, 42, 550-559. [CrossRef]

12. Luan, C.; Yao, X.; Shen, H.; Fu, J. Self-Sensing of Position-Related Loads in Continuous Carbon Fibers-Embedded 3D-Printed Polymer Structures Using Electrical Resistance Measurement. Sensors 2018, 18, 994. [CrossRef] [PubMed]

13. Wang, S.; Chung, D. Self-sensing of flexural strain and damage in carbon fiber polymer-matrix composite by electrical resistance measurement. Carbon 2006, 44, 2739-2751. [CrossRef]

14. Amenabar, I.; Mendikute, A.; Lopez-Arraiza, A.; Lizaranzu, M.; Aurrekoetxea, J. Comparison and analysis of non-destructive testing techniques suitable for delamination inspection in wind turbine blades. Compos. Part B Eng. 2011, 42, 1298-1305. [CrossRef]

15. Prakash, R.; Owston, C. Eddy-current method for the determination of lay-up order in cross-plied crfp laminates. Composites 1976, 7, 88-92. [CrossRef]

16. Haefner, B.; Berger, D. Design, data analysis and measurement uncertainty evaluation of an eddy-current sensor array for in-process metrology of carbon fiber reinforced plastics. CIRP Ann. 2019, 68, 539-542. [CrossRef]

17. Machado, M.A.; Antin, K.; Rosado, L.S.; Vilaça, P.; Santos, T.G. Contactless high-speed eddy current inspection of unidirectional carbon fiber reinforced polymer. Compos. Part B Eng. 2019, 168, 226-235. [CrossRef]

18. Megali, G.; Pellicano, D.; Cacciola, M.; Calcagno, S.; Versaci, M.; Morabito, F.C. EC modelling and enhancement signals in CFRP inspection. Prog. Electromagn. Res. M 2010, 14, 45-60. [CrossRef]

19. Swolfs, Y.; Verpoest, I.; Gorbatikh, L. A review of input data and modelling assumptions in longitudinal strength models for unidirectional fibre-reinforced composites. Compos. Struct. 2016, 150, 153-172. [CrossRef]

20. Park, J.; Okabe, T.; Takeda, N.; Curtin, W. Electromechanical modeling of unidirectional CFRP composites under tensile loading condition. Compos. Part A Appl. Sci. Manuf. 2002, 33, 267-275. [CrossRef] 